\title{
cccreative
}

ISSN 1855-3966 (printed edn.), ISSN 1855-3974 (electronic edn.)

\author{
ARS MATHEMATICA CONTEMPORANEA 18 (2020) 381-391 \\ https://doi.org/10.26493/1855-3974.2239.7f1 \\ (Also available at http://amc-journal.eu)
}

\section{The expansion of a chord diagram and the Genocchi numbers}

\author{
Tomoki Nakamigawa * \\ Department of Information Science, Shonan Institute of Technology, \\ Fujisawa, Kanagawa, Japan
}

Received 3 February 2020, accepted 21 April 2020, published online 24 October 2020

\begin{abstract}
A chord diagram $E$ is a set of chords of a circle such that no pair of chords has a common endvertex. Let $v_{1}, v_{2}, \ldots, v_{2 n}$ be a sequence of vertices arranged in clockwise order along a circumference. A chord diagram $\left\{v_{1} v_{n+1}, v_{2} v_{n+2}, \ldots, v_{n} v_{2 n}\right\}$ is called an $n$-crossing and a chord diagram $\left\{v_{1} v_{2}, v_{3} v_{4}, \ldots, v_{2 n-1} v_{2 n}\right\}$ is called an $n$-necklace. For a chord diagram $E$ having a 2-crossing $S=\left\{x_{1} x_{3}, x_{2} x_{4}\right\}$, the expansion of $E$ with respect to $S$ is to replace $E$ with $E_{1}=(E \backslash S) \cup\left\{x_{2} x_{3}, x_{4} x_{1}\right\}$ or $E_{2}=(E \backslash S) \cup\left\{x_{1} x_{2}, x_{3} x_{4}\right\}$. Beginning from a given chord diagram $E$ as the root, by iterating chord expansions in both ways, we have a binary tree whose all leaves are nonintersecting chord diagrams. Let $\mathcal{N C D}(E)$ be the multiset of the leaves. In this paper, the multiplicity of an $n$-necklace in $\mathcal{N C D}(E)$ is studied. Among other results, it is shown that the multiplicity of an $n$-necklace generated from an $n$-crossing equals the Genocchi number when $n$ is odd and the median Genocchi number when $n$ is even.
\end{abstract}

Keywords: Chord diagram, chord expansion, Genocchi number, Seidel triangle.

Math. Subj. Class. (2020): 05A15, 05A10

\section{Introduction}

A set of chords of a circle is called a chord diagram, if they have no common endvertex. If a chord diagram consists of a set of $n$ mutually crossing chords, it is called an $n$-crossing. A 2-crossing is simply called a crossing as well. If a chord diagram contains no crossing, it is called nonintersecting.

Let $V$ be a set of $2 n$ vertices on a circle, and let $E$ be a chord diagram of order $n$, where each chord has endvertices of $V$. In this situation, $V$ is called a support of

*This work was supported by JSPS KAKENHI Grant Number 19K03607.
E-mail address: nakami@ info.shonan-it.ac.jp (Tomoki Nakamigawa)

() (i) This work is licensed under https://creativecommons.org/licenses/by/4.0/ 
$E$. We denote the family of all chord diagrams having $V$ as a support by $\mathcal{C D}(V)$. Let $x_{1}, x_{2}, x_{3}, x_{4} \in V$ be placed on a circle in clockwise order. Let $E \in \mathcal{C D}(V)$. For a crossing $S=\left\{x_{1} x_{3}, x_{2} x_{4}\right\} \subset E$, let $S_{1}=\left\{x_{2} x_{3}, x_{4} x_{1}\right\}$, and $S_{2}=\left\{x_{1} x_{2}, x_{3} x_{4}\right\}$. The expansion of $E$ with respect to $S$ is defined as a replacement of $E$ with $E_{1}=(E \backslash S) \cup S_{1}$ or $E_{2}=(E \backslash S) \cup S_{2}$ (see Figure 1).

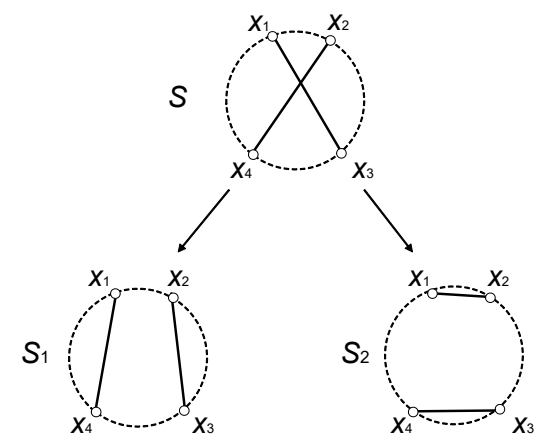

Figure 1: The expansion of a chord diagram with respect to a 2 -crossing $S$. Other chords except those in $S$ are not shown.

Let $E \in \mathcal{C D}(V)$ be a chord diagram. Form a binary tree as follows. Begin with $E$ as the root, arbitrarily choose a crossing of $E$, and expand $E$ in both ways, adding the results as children of $E$. Choose crossings in each child if any exists, expand them each in both ways, and repeat the procedure until all leaves are nonintersecting. This procedure terminates and the multiset of leaves is independent of the choices made at each step ([14]). Let us denote the multiset of nonintersecting chord diagrams generated from $E$ by $\mathcal{N} \mathcal{C D}(E)$. For a chord diagram $E \in \mathcal{C D}(V)$, let us define the chord expansion number $f(E)$ as the cardinality of $\mathcal{N C D}(E)$ as a multiset.

For a chord diagram $E$, the circle graph, also called the interlace graph $G_{E}$ of $E$, is a graph such that a vertex of $G_{E}$ corresponds to a chord of $E$ and two vertices of $G_{E}$ are joined by an edge if their corresponding chords of $E$ are mutually crossing. We say that two chord diagrams $E_{1}$ and $E_{2}$ with a common support are isomorphic if $G_{E_{1}}$ and $G_{E_{2}}$ are isomorphic as graphs. It is proved that $f(E)$ equals $t\left(G_{E} ; 2,-1\right)$, where $t(G ; x, y)$ is the Tutte polynomial of a graph $G$ ([15]).

In the case $E$ is an $n$-crossing $C_{n}$, its associated circle graph is a complete graph $K_{n}$ with $n$ vertices. In [13], Merino proved that $t\left(K_{n} ; 2,-1\right)=E u l_{n+1}$ for $n \geq 1$, where $(E u l)_{n \geq 1}=(1,1,2,5,16,61,272, \ldots)$ is the Euler number. Hence, we have $f\left(C_{n}\right)=$ $E u l_{n+1}$ for $n \geq 1$. See also [12] for the evaluation of $t(G ; 2,-1)$ for a graph $G$.

For two nonnegative integers $k$ and $n$ with $k \leq n$, we define $A(n, k)$ as a chord diagram of order $n+1$, in which there is an $n$-crossing $E_{0}$ with an extra chord $e$ such that $e$ crosses exactly $k$ chords of $E_{0}$. (See Figure 2.) Note that $A(n-1, n-1)$ is simply an $n$-crossing, and that $A(n, 0)$ is a union of an $n$-crossing and an isolated chord.

Let us denote $\{1,2, \ldots, n\}$ by $[n]$. A permutation $\sigma$ on $[n]$ is called an alternating permutation if $(\sigma(i)-\sigma(i-1))(\sigma(i+1)-\sigma(i))<0$ for $2 \leq i \leq n-1$. An alternating permutation $\sigma$ is called an up-down permutation (resp. down-up permutation) if $\sigma(1)<$ $\sigma(2)$ (resp. $\sigma(1)>\sigma(2)$ ). For $0 \leq k \leq n$, the Entringer number Ent ${ }_{n, k}$ is defined as the number of down-up permutations on $[n+1]$ with the first term $k+1$ ([11]). For $n \geq 1$, 

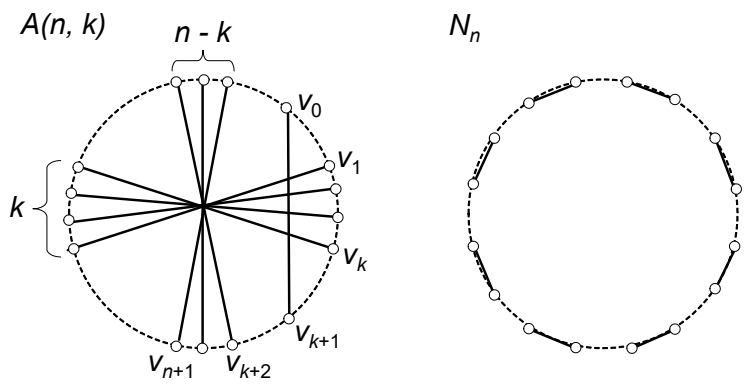

Figure 2: $A(n, k)$ with $n=7$ and $k=4$ (left), and $N_{n}$ with $n=8$ (right).

$E n t_{n+1,1}$ equals $E u l_{n}$, the number of all down-up permutations on [n]. In [14], it is proved that $f(A(n, k))=E_{n+2, k+1}$.

For a chord diagram $E$ and for a nonintersecting chord diagram $F$ with a common support, let us denote the multiplicity of $F$ in $\mathcal{N C D}(E)$ by $m(E, F)$. For a nonintersecting chord diagram $E$, a chord $e \in E$ is called an ear, if there is no other chord of $E$ on at least one side of $e$. In [15], it is shown that for an $n$-crossing $C_{n}$ and a nonintersecting chord diagram $F$ with a common support, $m\left(C_{n}, F\right)=1$ if and only if $F$ has at most 3 ears. A nonintersecting chord diagram $E$ with $n$ chords is called an $n$-necklace, denoted by $N_{n}$, if all chords of $E$ are ears. (See Figure 2.) The main purpose of the paper is to show that $m\left(C_{n}, N_{n}\right)$ equals the Genocchi number when $n$ is odd and the median Genocchi number when $n$ is even. The Genocchi numbers and the median Genocchi numbers will be introduced in the following section.

Recently, Bigeni showed a relation between a weight system of $\mathrm{sl}_{2}$ of chord diagrams and the median Genocchi numbers ([2]). In Definition 1 of [2], followed from [3], a weight system of $\mathrm{sl}_{2}$ is defined inductively by applying an operation for chord diagrams. The operation and the chord expansion are closely related to each other, although our main results in the paper do not seem directly followed from the results in [2].

The rest of this paper is organized as follows. In Section 2, the Genocchi numbers and the median Genocchi numbers are introduced. In Section 3, the main results of the paper are proved. In Section 4, another combinatorial interpretation for the multiplicity of $n$-necklaces is exhibited. Finally, in Section 5, some open problems are discussed.

\section{The Genocchi numbers and the median Genocchi numbers}

According to [10], but with slightly different indices, let us recursively define the entry $S(n, k)$ in row $n \geq 1$ and column $k \geq 0$ of the Seidel triangle ([17]):

$$
\begin{aligned}
S(1,1) & =1, \\
S(n, k) & =0 \quad \text { for } k=0 \text { or } n \leq 2(k-1), \\
S(2 n, k) & =\sum_{i \geq k} S(2 n-1, i) \quad \text { for } 1 \leq k \leq n, \\
S(2 n+1, k) & =\sum_{i \leq k} S(2 n, i) \quad \text { for } 1 \leq k \leq n+1 .
\end{aligned}
$$


Table 1: The Seidel triangle $S(n, k)$.

\begin{tabular}{r|rrrrr}
$n \backslash k$ & 1 & 2 & 3 & 4 & 5 \\
\hline 1 & 1 & & & & \\
2 & 1 & & & & \\
3 & 1 & 1 & & & \\
4 & 2 & 1 & & & \\
5 & 2 & 3 & 3 & & \\
6 & 8 & 6 & 3 & & \\
7 & 8 & 14 & 17 & 17 & \\
8 & 56 & 48 & 34 & 17 & \\
9 & 56 & 104 & 138 & 155 & 155 \\
10 & 608 & 552 & 448 & 310 & 155
\end{tabular}

(See Table 1.) By the equations (2.1) and (2.2), we have the following recurrence relations.

$$
\begin{aligned}
S(2 n, k)=S(2 n-1, k)+S(2 n, k+1) & \text { for } 1 \leq k \leq n, \\
S(2 n+1, k)=S(2 n, k)+S(2 n+1, k-1) & \text { for } 1 \leq k \leq n+1 .
\end{aligned}
$$

The Genocchi numbers (or Genocchi numbers of the first kind) $G(2 n)$ are defined as $S(2 n-1, n)$, the numbers on the right edge of the Seidel triangle, and the median Genocchi numbers (or Genocchi numbers of the second kind) $H(2 n+1)$ are defined as $S(2 n+2,1)$, the numbers on the left edge of the Seidel triangle. Note that $(G(2 n))_{n \geq 1}=$ $(1,1,3,17,155, \ldots)$ and $(H(2 n+1))_{n \geq 0}=(1,2,8,56,608, \ldots)$.

Combinatorial properties of the Genocchi numbers have been extensively studied ([1, $4,5,6,7,8,9,10,16,19])$. It is known that the Genocchi number $G(2 n)$ counts the number of permutations $\sigma$ on $[2 n-1]$ such that $\sigma(i)<\sigma(i+1)$ if $\sigma(i)$ is odd, and $\sigma(i)>\sigma(i+1)$ if $\sigma(i)$ is even ([6]). It is also known that the median Genocchi number $H(2 n+1)$ counts the number of permutations $\sigma$ on $[2 n+1]$ such that $\sigma(i)>i$ if $i$ is odd and $i \neq 2 n+1$, and $\sigma(i)<i$ if $i$ is even ([6]).

In the on-line encyclopedia of integer sequences [18], we can find more information for the sequences A001469 (Genocchi numbers), A005439 (median Genocchi numbers), A099960 (An interleaving of the Genocchi numbers of the first and second kind) and A014781 (Seidel triangle).

\section{Main results}

Our aim is to show a new combinatorial interpretation for the values of the Seidel triangle by using chord expansions.

Let $v_{0}, v_{1}, \ldots, v_{2 n+1}$ be a sequence of vertices in clockwise order along a circumference. Let $V=\left\{v_{i}: 0 \leq i \leq 2 n+1\right\}$. As one of chord diagrams $E \in \mathcal{C D}(V)$ isomorphic to $A(n, k)$, introduced in the previous section, we have $E=\left\{v_{0} v_{k+1}\right\} \cup\left\{v_{i} v_{n+i+1}\right.$ : $1 \leq i \leq k\} \cup\left\{v_{i} v_{n+i}: k+2 \leq i \leq n+1\right\}$. (See Figure 2.) Now let us define $(n+1)$ necklaces $N_{n+1, k}^{+}$and $N_{n+1, k}^{-} \in \mathcal{C D}(V)$ such that $N_{n+1, k}^{+}$contains an ear $v_{k} v_{k+1}$ and $N_{n+1, k}^{-}$contains an ear $v_{k+1} v_{k+2}$. The values of $m\left(A(n, k), N_{n+1, k}^{+}\right)$for $n$ and $k$ small are shown in Table 2. 
Table 2: $m\left(A(n, k), N_{n+1, k}^{+}\right)$for $0 \leq k \leq n \leq 8$.

\begin{tabular}{r|rrrrrrrrr}
$n \backslash k$ & 0 & 1 & 2 & 3 & 4 & 5 & 6 & 7 & 8 \\
\hline 0 & 1 & & & & & & & & \\
1 & 1 & 1 & & & & & & & \\
2 & 1 & 1 & 1 & & & & & & \\
3 & 1 & 1 & 2 & 2 & & & & & \\
4 & 2 & 2 & 3 & 3 & 3 & & & & \\
5 & 3 & 3 & 6 & 6 & 8 & 8 & & & \\
6 & 8 & 8 & 14 & 14 & 17 & 17 & 17 & & \\
7 & 17 & 17 & 34 & 34 & 48 & 48 & 56 & 56 & \\
8 & 56 & 56 & 104 & 104 & 138 & 138 & 155 & 155 & 155
\end{tabular}

Let us define $b_{n, k}^{+}=m\left(A(n, k), N_{n+1, k}^{+}\right)$and $b_{n, k}^{-}=m\left(A(n, k), N_{n+1, k}^{-}\right)$. We also simply denote $b_{n, k}^{+}$by $b_{n, k}$. The main result of the paper is the following theorem.

Theorem 3.1. Let $n \geq 1$. Then we have

$$
b_{2 n-1, k}=S(2 n, n-\lfloor k / 2\rfloor) \quad \text { for } 0 \leq k \leq 2 n-1
$$

and

$$
b_{2 n, k}=S(2 n+1,\lfloor k / 2\rfloor+1) \quad \text { for } 0 \leq k \leq 2 n .
$$

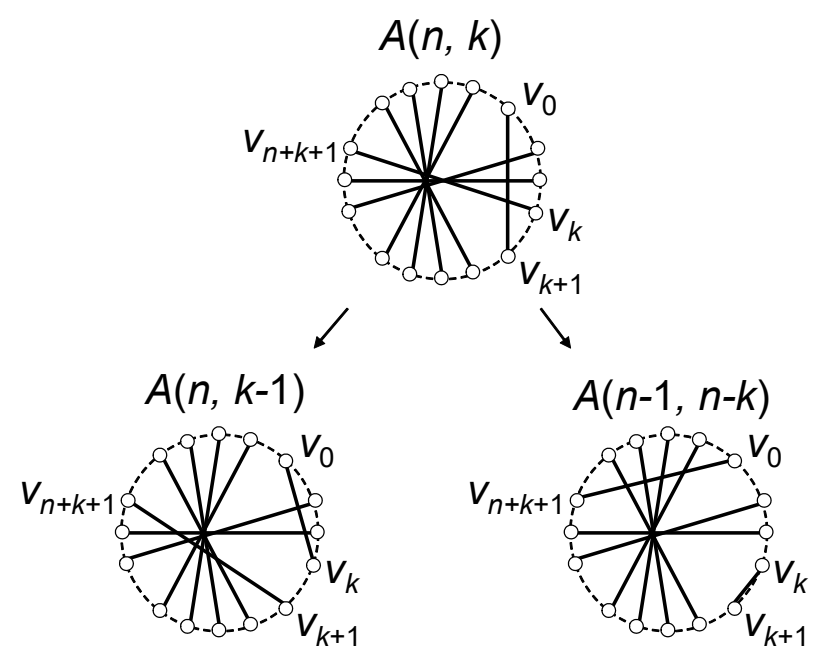

Figure 3: A chord expansion of $A(n, k)$ with respect to $\left\{v_{0} v_{k+1}, v_{k} v_{n+k+1}\right\}$ with $n=7$ and $k=3$.

Firstly, we show a relation between $b_{n, k}^{-}$and $b_{n, k}^{+}$.

Lemma 3.2. $b_{n, k}^{-}=b_{n, k-1}^{+}$for $1 \leq k \leq n$. 
Proof. Let $E$ be a chord diagram isomorphic to $A(n, k)$, as shown in Figure 3. By the chord expansion of $E$ with respect to $\left\{v_{0} v_{k+1}, v_{k} v_{n+k+1}\right\}$, we have two successors $E_{1}$ and $E_{2}$, which are isomorphic to $A(n, k-1)$ and $A(n-1, n-k)$, respectively. Since $E_{2}$ contains a chord $v_{k} v_{k+1}$, it does not generate $N_{n+1, k}^{-}$. Furthermore, since $N_{n+1, k}^{-}$is a necklace having a chord $v_{k-1} v_{k}$, we have $b_{n, k}^{-}=m\left(A(n, k), N_{n+1, k}^{-}\right)=m\left(A(n, k-1), N_{n+1, k-1}^{+}\right)=$ $b_{n, k-1}^{+}$, as required.

In order to prove Theorem 3.1, let us show a recurrence relation for $b_{n, k}$.

Lemma 3.3. We have $b_{0,0}=1$ and for $n \geq 1$, we have

$$
\begin{aligned}
& b_{n, 0}=b_{n, 1}=b_{n-1, n-1}, \\
& b_{n, k}= \begin{cases}b_{n, k-2}+b_{n-1, n-k} & \text { for } 2 \leq k \leq n \text { and } n \text { is odd }, \\
b_{n, k-2}+b_{n-1, n-k-1} & \text { for } 2 \leq k \leq n-1 \text { and } n \text { is even, }\end{cases} \\
& b_{n, n}=b_{n, n-2} \text { for } n \text { is even. }
\end{aligned}
$$

Proof. When $k=0,1$ or $n$, equations $b_{n, 0}=b_{n, 1}=b_{n-1, n-1}$ can be proved easily. Let us consider the case $2 \leq k \leq n$. As in the proof of Lemma 3.2, we use the expansion of $A(n, k)$ with respect to $\left\{v_{0} v_{k+1}, v_{k} v_{n+k+1}\right\}$.

If $n$ is odd, we have

$$
\begin{aligned}
b_{n, k}^{+} & =b_{n, k-1}^{-}+b_{n-1, n-k}^{+} \\
& =b_{n, k-2}^{+}+b_{n-1, n-k}^{+}
\end{aligned}
$$

If $n$ is even and $k<n$, we have

$$
\begin{aligned}
b_{n, k}^{+} & =b_{n, k-1}^{-}+b_{n-1, n-k}^{-} \\
& =b_{n, k-2}^{+}+b_{n-1, n-k-1}^{+}
\end{aligned}
$$

Finally, if $n$ is even and $k=n$, since $b_{n-1,0}^{-}=0$, we have

$$
\begin{aligned}
b_{n, n}^{+} & =b_{n, n-1}^{-}+b_{n-1,0}^{-} \\
& =b_{n, n-2}^{+},
\end{aligned}
$$

as needed.

Proof of Theorem 3.1. We proceed by induction on $n$ and $k$. For (3.1) with $n=1$, we have $b_{1,0}=1$ and $b_{1,1}=1$. On the other hand, we have $S(2,1)=1$. For (3.2) with $n=1$, we have $b_{2,0}=1, b_{2,1}=1$ and $b_{2,2}=1$. On the other hand, we have $S(3,1)=S(3,2)=1$.

Let $n \geq 2$. For $k=0$, we have

$$
\begin{aligned}
b_{2 n-1,0} & =b_{2 n-2,2 n-2} \\
& =S(2 n-1, n) \\
& =S(2 n, n),
\end{aligned}
$$


and

$$
\begin{aligned}
b_{2 n, 0} & =b_{2 n-1,2 n-1} \\
& =S(2 n, 1) \\
& =S(2 n+1,1) .
\end{aligned}
$$

For $k=1$, we have

$$
\begin{aligned}
b_{2 n-1,1} & =b_{2 n-1,0} \\
& =S(2 n, n),
\end{aligned}
$$

and

$$
\begin{aligned}
b_{2 n, 1} & =b_{2 n, 0} \\
& =S(2 n+1,1) .
\end{aligned}
$$

For (3.1) with $2 \leq k \leq 2 n-1$, we have

$$
\begin{aligned}
b_{2 n-1, k} & =b_{2 n-1, k-2}+b_{2 n-2,2 n-1-k} \\
& =S(2 n, n-\lfloor(k-2) / 2\rfloor)+S(2 n-1,\lfloor(2 n-1-k) / 2\rfloor+1) \\
& =S(2 n, n+1-\lfloor k / 2\rfloor)+S(2 n-1, n-\lfloor k / 2\rfloor) \\
& =S(2 n, n-\lfloor k / 2\rfloor),
\end{aligned}
$$

and for (3.2) with $2 \leq k \leq 2 n-1$, we have

$$
\begin{aligned}
b_{2 n, k} & =b_{2 n, k-2}+b_{2 n-1,2 n-1-k} \\
& =S(2 n+1,\lfloor(k-2) / 2\rfloor+1)+S(2 n, n-\lfloor(2 n-1-k) / 2\rfloor) \\
& =S(2 n+1,\lfloor k / 2\rfloor)+S(2 n, 1+\lfloor k / 2\rfloor) \\
& =S(2 n+1,1+\lfloor k / 2\rfloor),
\end{aligned}
$$

and for (3.2) with $k=2 n$, we have

$$
\begin{aligned}
b_{2 n, 2 n} & =b_{2 n, 2 n-2} \\
& =S(2 n+1, n) \\
& =S(2 n+1, n+1) .
\end{aligned}
$$

By Theorem 3.1, we have the following corollary.

Corollary 3.4. $m\left(C_{2 n}, N_{2 n}\right)=H(2 n-1)$ and $m\left(C_{2 n-1}, N_{2 n-1}\right)=G(2 n)$ for $n \geq 1$.

Proof. By Theorem 3.1, we have $m\left(C_{2 n}, N_{2 n}\right)=b_{2 n-1,2 n-1}=S(2 n, 1)=H(2 n-1)$, and $m\left(C_{2 n-1}, N_{2 n-1}\right)=b_{2 n-2,2 n-2}=S(2 n-1, n)=G(2 n)$.

\section{Multiplicity of an $N$-necklace and the number of perfect matchings of an associated graph}

In this section, we will exhibit a combinatorial interpretation of $m\left(E, N_{n}\right)$ for a given chord diagram $E$. For a set $V$ of vertices on the circumference, $\mathcal{C}(V)$ denotes the set of all 
chords whose endvertices are in $V$. A Ptolemy weight $w$ on $\mathcal{C}(V)$ is defined as a function that satisfies

$$
w\left(x_{1} x_{3}\right) w\left(x_{2} x_{4}\right)=w\left(x_{2} x_{3}\right) w\left(x_{1} x_{4}\right)+w\left(x_{1} x_{2}\right) w\left(x_{3} x_{4}\right)
$$

for all vertices $x_{1}, x_{2}, x_{3}, x_{4} \in V$ placed along the circle. If $w(e)$ is the Euclidean length of a chord $e$, then (4.1) holds by the Ptolemy's theorem in Euclidean geometry. Let $w$ be a Ptolemy weight on $\mathcal{C}(V)$. If a chord diagram $E \in \mathcal{C D}(V)$ has a 2-crossing $S$, by the chord expansion of $E$ with respect to $S$, we have two successors $E_{1}$ and $E_{2}$. Then by (4.1), we have

$$
\prod_{e \in E} w(e)=\prod_{e \in E_{1}} w(e)+\prod_{e \in E_{2}} w(e) .
$$

We denote the left-hand side of (4.2) by $w(E)$. By iterating chord expansions with (4.2), we have

$$
w(E)=\sum_{F \in \mathcal{N} \mathcal{C D}(E)} w(F) .
$$

Let $V=\left\{v_{1}, v_{2}, \ldots, v_{2 n}\right\}$, where $v_{1}, v_{2}, \ldots, v_{2 n}$ are placed along the circumference in this order. A Ptolemy weight $w$ on $\mathcal{C}(V)$ is called rectilinear if $w\left(v_{i} v_{j}\right)=$ $\sum_{i \leq k<j} w\left(v_{k} v_{k+1}\right)$ for all $1 \leq i<j \leq 2 n$. For example, if the vertices are placed on a straight line and the weight $w\left(v_{i} v_{j}\right)$ is defined as the Euclidean distance between $v_{i}$ and $v_{j}$, then $w$ is indeed a rectilinear Ptolemy weight.

In order to analyze $m\left(E, N_{n}\right)$, let us consider the rectilinear Ptolemy weight $w$ on $\mathcal{C}(V)$ such that $w\left(v_{2 k-1} v_{2 k}\right)=x_{k}$ for $1 \leq k \leq n$ and $w\left(v_{2 k} v_{2 k+1}\right)=0$ for $1 \leq k \leq n-1$. In this weight, since for every chord $e, w(e)$ corresponds to a first degree polynomial of a multiple variables $x_{1}, x_{2}, \ldots, x_{n}$ or $w(e)=0$, for all chord diagrams $E, w(E)$ is a homogeneous polynomial of degree $n$ or $w(E)=0$. From this point until the end of this section, we fix this weight. Let us define an $n$-necklace $N_{n}=\left\{v_{2 k-1} v_{2 k}: 1 \leq k \leq n\right\}$.

Lemma 4.1. In the rectilinear Ptolemy weight $w$ as defined in the above, for a chord diagram $E, m\left(E, N_{n}\right)$ equals the coefficient of $x_{1} x_{2} \ldots x_{n}$ of the polynomial $w(E)$.

Proof. Since $w\left(N_{n}\right)=x_{1} x_{2} \ldots x_{n}$, what we need to show is that if $F \in \mathcal{N C D}(E) \backslash\left\{N_{n}\right\}$, a polynomial $w(F)$ contains no monomial $x_{1} x_{2} \ldots x_{n}$. Suppose to a contradiction that $F \in \mathcal{N C D}(E) \backslash\left\{N_{n}\right\}$ and $F$ has a monomial $x_{1} x_{2} \ldots x_{n}$. Since $F \neq N_{n}$, there exists a chord $v_{2 k-1} v_{2 \ell}$ of $F$ with $1 \leq k<\ell \leq n$ such that $\ell-k \geq 1$ is maximal. Then the two variables $x_{k}$ and $x_{\ell}$ do not appear together in the weight of any chord of $F$, otherwise such a chord would either intersect $v_{2 k-1} v_{2 \ell}$ or contradict $\ell-k$ being maximal. It follows that the product $x_{k} x_{\ell}$ never appears in $w(F)$. This contradicts to that $w(F)$ contains a monomial $x_{1} x_{2} \ldots x_{n}$.

For a chord diagram $E$ having $n$ chords $e_{1}, e_{2}, \ldots, e_{n}$ with the rectilinear Ptolemy weight $w$ as defined in the above, let us define a balanced bipartite graph $G(E, X)$ with partite sets $A=\left\{a_{1}, a_{2}, \ldots, a_{n}\right\}$ and $B=\left\{b_{1}, b_{2}, \ldots, b_{n}\right\}$ as follows. For $1 \leq i \leq n$ and $1 \leq j \leq n, a_{i}$ and $b_{j}$ are adjacent if and only if a polynomial $w\left(e_{i}\right)$ contains a monomial $x_{j}$.

Theorem 4.2. For a chord diagram $E$ with $n$ chords and its associated balanced bipartite graph $G(E, X)$ as defined in the above, $m\left(E, N_{n}\right)$ equals the number of perfect matchings of $G(E, X)$. 
Proof. We have $w(E)=\prod_{e \in E} w(e)$, and for all chords $e, w(e)=0$ or $w(e)=x_{i}+$ $x_{i+1}+\cdots+x_{j}$ for some $1 \leq i \leq j \leq n$. Hence, the coefficient of $x_{1} x_{2} \ldots x_{n}$ of $w(E)$, which is $m\left(E, N_{n}\right)$ by Lemma 4.1 , is the number of possible combinations to choose a variable $x \in X$ from each $w(e)$ without repetition. This is the number of perfect matchings of $G(E, X)$.

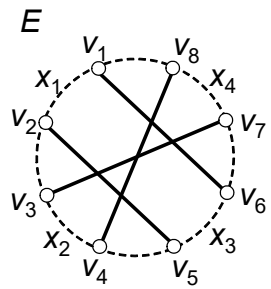

$G(E, X)$

\begin{tabular}{|l|l|l|l|l|}
\hline & $x_{1}$ & $x_{2}$ & $x_{3}$ & $x_{4}$ \\
\hline$e_{1}$ & 1 & 1 & 1 & 0 \\
\hline$e_{2}$ & 0 & 1 & 0 & 0 \\
\hline$e_{3}$ & 0 & 1 & 1 & 0 \\
\hline$e_{4}$ & 0 & 0 & 1 & 1 \\
\hline
\end{tabular}

Figure 4: A chord diagram $E$ (left) and its biadjacency matrix of a corresponding bipartite graph $G(E, X)$ (right).

Example 4.3. Let $n=4$. Let $V=\left\{v_{i}: 1 \leq i \leq 2 n\right\}$, where $v_{1}, v_{2}, \ldots, v_{2 n}$ are placed on the circumference in this order. Let us consider a rectilinear Ptolemy weight $w$ on $\mathcal{C}(V)$ such that $w\left(v_{2 i-1} v_{2 i}\right)=x_{i}$ for $1 \leq i \leq n$ and $w\left(v_{2 i} v_{2 i+1}\right)=0$ for $1 \leq i \leq n-1$. Let $E=\left\{e_{i}: 1 \leq i \leq 4\right\}$ be a chord diagram, where $e_{1}=v_{1} v_{6}, e_{2}=v_{2} v_{5}, e_{3}=v_{3} v_{7}, e_{4}=$ $v_{4} v_{8}$. (See Figure 4.) Since

$$
w(E)=\prod_{1 \leq i \leq n} w\left(e_{i}\right)=\left(x_{1}+x_{2}+x_{3}\right) x_{2}\left(x_{2}+x_{3}\right)\left(x_{3}+x_{4}\right),
$$

the coefficient of $x_{1} x_{2} x_{3} x_{4}$ of $w(E)$ is 1 , and the number of perfect matchings of $G(E, X)$ is also 1 . Hence, we have $m\left(E, N_{n}\right)=1$.

By Corollary 3.4 and Theorem 4.2 for $n$-crossings $C_{n}$, we have the following corollary.

Corollary 4.4. The number of perfect matchings of the following bipartite graphs $G$ and $H$ corresponds to Genocchi numbers $G(2 n)$ and median Genocchi numbers $H(2 n-1)$ as follows:

$$
\begin{aligned}
& V(G)=E \cup X, \quad \text { where } E=\left\{e_{1}, e_{2}, \ldots, e_{2 n-1}\right\}, X=\left\{x_{1}, x_{2}, \ldots, x_{2 n-1}\right\}, \\
& E(G)=\left\{e_{i} x_{j}: 1 \leq i \leq 2 n-1,\lfloor i / 2\rfloor+1 \leq j \leq\lfloor(i-1) / 2\rfloor+n\right\} . \\
& V(H)=E \cup X, \quad \text { where } E=\left\{e_{1}, e_{2}, \ldots, e_{2 n}\right\}, X=\left\{x_{1}, x_{2}, \ldots, x_{2 n}\right\}, \\
& E(H)=\left\{e_{i} x_{j}: 1 \leq i \leq 2 n,\lfloor i / 2\rfloor+1 \leq j \leq\lfloor i / 2\rfloor+n\right\} .
\end{aligned}
$$

Example 4.5. As shown in Figure 5,

$$
w\left(C_{6}\right)=\left(x_{1}+x_{2}+x_{3}\right)\left(x_{2}+x_{3}+x_{4}\right)^{2}\left(x_{3}+x_{4}+x_{5}\right)^{2}\left(x_{4}+x_{5}+x_{6}\right) .
$$

The coefficient of $x_{1} x_{2} x_{3} x_{4} x_{5} x_{6}$ of $w\left(C_{6}\right)$ is 8 , and the number of perfect matchings of $G\left(C_{6}, X\right)$ is also 8. Hence, we have $H(5)=m\left(C_{6}, N_{6}\right)=8$.

$$
\begin{array}{r}
w\left(C_{7}\right)=\left(x_{1}+x_{2}+x_{3}+x_{4}\right)\left(x_{2}+x_{3}+x_{4}\right)\left(x_{2}+x_{3}+x_{4}+x_{5}\right)\left(x_{3}+x_{4}+x_{5}\right) \\
\left(x_{3}+x_{4}+x_{5}+x_{6}\right)\left(x_{4}+x_{5}+x_{6}\right)\left(x_{4}+x_{5}+x_{6}+x_{7}\right) .
\end{array}
$$



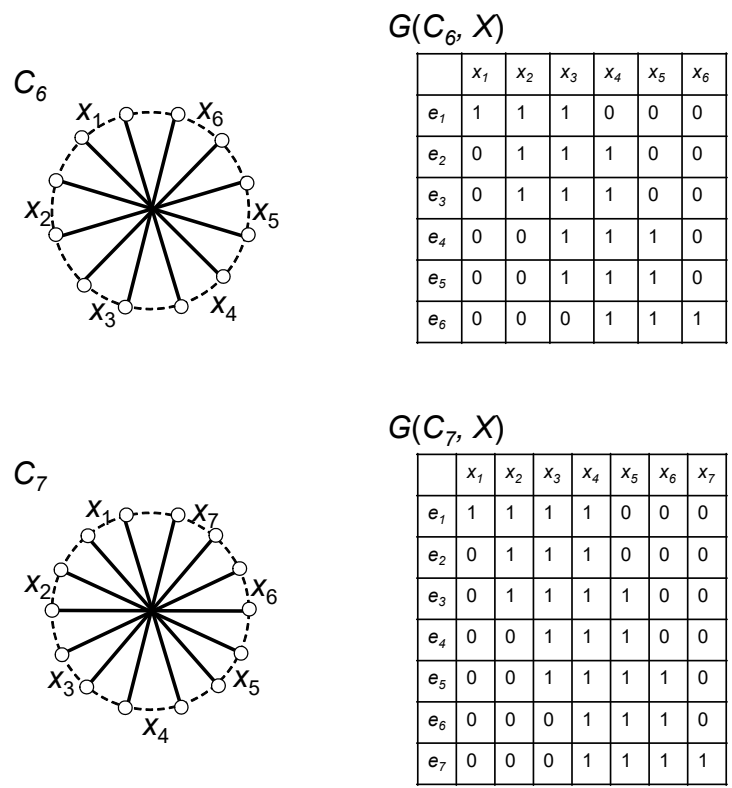

Figure 5: $n$-crossings (upper left, lower left) and their biadjacency matrices of corresponding bipartite graphs $G\left(C_{n}, X\right)$ (upper right, lower right).

The coefficient of $x_{1} x_{2} x_{3} x_{4} x_{5} x_{6} x_{7}$ of $w\left(C_{7}\right)$ is 17 , and the number of perfect matchings of $G\left(C_{7}, X\right)$ is also 17 . Hence, we have $G(8)=m\left(C_{7}, N_{7}\right)=17$.

\section{Further discussions}

There are a lot of unknown things for the multiplicity in $\mathcal{N C D}(E)$. One ambitious problem is to find a formula for $m(E, F)$ in general.

In Section 4, we represent $m\left(E, N_{n}\right)$ by the number of perfect matchings of a corresponding bipartite graph. It is interesting if we can find an efficient method to calculate the number of perfect matchings in a graph of this kind.

As is shown in [15], there is a relation between the chord expansion number and the evaluation of the Tutte polynomial at the point $(2,-1)$. As a future research subject, it is considered to find a relation between the multiplicity $m(E, F)$ in general, or $m\left(E, N_{n}\right)$, and some counting polynomials of graphs.

\section{References}

[1] A. Bigeni, Combinatorial interpretations of the Kreweras triangle in terms of subset tuples, Electron. J. Combin. 25 (2018), \#P4.44 (11 pages), doi:10.37236/7531.

[2] A. Bigeni, A generalization of the Kreweras triangle through the universal $\mathrm{sl}_{2}$ weight system, J. Comb. Theory Ser. A 161 (2019), 309-326, doi:10.1016/j.jcta.2018.08.005.

[3] S. V. Chmutov and A. N. Varchenko, Remarks on the Vassiliev knot invariants coming from $\mathrm{sl}_{2}$, Topology 36 (1997), 153-178, doi:10.1016/0040-9383(95)00071-2. 
[4] M. Domaratzki, Combinatorial interpretations of a generalization of the Genocchi numbers, J. Integer Seq. 7 (2004), Article 04.3.6 (11 pages), https: / / cs . uwaterloo.ca/ journals/JIS/VOL7/Domaratzki/doma23.html.

[5] D. Dumont, Sur une conjecture de Gandhi concernant les nombres de Genocchi, Discrete Math. 1 (1972), 321-327, doi:10.1016/0012-365x(72)90039-8.

[6] D. Dumont, Interprétations combinatoires des nombres de Genocchi, Duke Math. J. 41 (1974), 305-318, doi:10.1215/s0012-7094-74-04134-9.

[7] D. Dumont and D. Foata, Une propriété de symétrie des nombres de Genocchi, Bull. Soc. Math. France 104 (1976), 433-451, doi:10.24033/bsmf.1839.

[8] D. Dumont and A. Randrianarivony, Dérangements et nombres de Genocchi, Discrete Math. 132 (1994), 37-49, doi:10.1016/0012-365x(94)90230-5.

[9] D. Dumont and G. Viennot, A combinatorial interpretation of the Seidel generation of Genocchi numbers, in: J. Srivastava (ed.), Combinatorial Mathematics, Optimal Designs and Their Applications, Elsevier, volume 6 of Annals of Discrete Mathematics, pp. 77-87, 1980, doi: 10.1016/S0167-5060(08)70696-4.

[10] R. Ehrenborg and E. Steingrímsson, Yet another triangle for the Genocchi numbers, European J. Combin. 21 (2000), 593-600, doi:10.1006/eujc.1999.0370.

[11] R. C. Entringer, A combinatorial interpretation of the Euler and Bernoulli numbers, Nieuw Arch. Wisk. 14 (1966), 241-246.

[12] A. J. Goodall, C. Merino, A. de Mier and M. Noy, On the evaluation of the Tutte polynomial at the points $(1,-1)$ and $(2,-1)$, Ann. Comb. 17 (2013), 311-332, doi:10.1007/ s00026-013-0180-y.

[13] C. Merino, The number of 0-1-2 increasing trees as two different evaluations of the Tutte polynomial of a complete graph, Electron. J. Combin. 15 (2008), \#N28 (5 pages), doi:10.37236/ 903.

[14] T. Nakamigawa, Expansions of a chord diagram and alternating permutations, Electron. J. Combin. 23 (2016), \#P1.7 (8 pages), doi:10.37236/5120.

[15] T. Nakamigawa and T. Sakuma, The expansion of a chord diagram and the Tutte polynomial, Discrete Math. 341 (2018), 1573-1581, doi:10.1016/j.disc.2018.02.015.

[16] J. Riordan and P. R. Stein, Proof of a conjecture on Genocchi numbers, Discrete Math. 5 (1973), 381-388, doi:10.1016/0012-365x(73)90131-3.

[17] L. Seidel, Über eine einfache entstehungsweise der Bernoullischen zahlen und einiger verwandten reihen, Sitzungsber. Münch. Akad. 4 (1877), 157-187, http: / / publikationen. badw. de/de/003384831.

[18] N. J. A. Sloane (ed.), The On-Line Encyclopedia of Integer Sequences, published electronically at https://oeis.org.

[19] G. Viennot, Interprétations combinatoires des nombres d'Euler et de Genocchi, in: Seminar on Number Theory, 1981/1982, Université de Bordeaux I, Talence, Exp. No. 11 (94 pages), 1982, held at the Université de Bordeaux I, Talence, 1981/1982. 